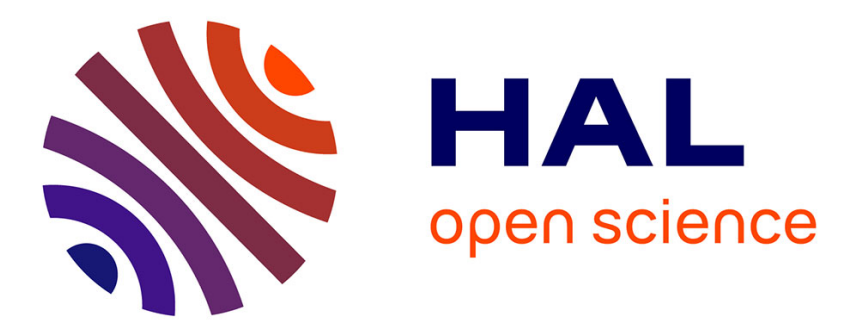

\title{
The bifurcation structure of viscous steady axisymmetric vortex breakdown with open lateral boundaries
}

Elena Vyazmina, Joseph W. Nichols, Jean-Marc Chomaz, Peter J. Schmid

\section{To cite this version:}

Elena Vyazmina, Joseph W. Nichols, Jean-Marc Chomaz, Peter J. Schmid. The bifurcation structure of viscous steady axisymmetric vortex breakdown with open lateral boundaries. Physics of Fluids, 2009, 21 (7), pp.074107. 10.1063/1.3176476 . hal-01002602

HAL Id: hal-01002602

https://hal-polytechnique.archives-ouvertes.fr/hal-01002602

Submitted on 7 Jul 2014

HAL is a multi-disciplinary open access archive for the deposit and dissemination of scientific research documents, whether they are published or not. The documents may come from teaching and research institutions in France or abroad, or from public or private research centers.
L'archive ouverte pluridisciplinaire HAL, est destinée au dépôt et à la diffusion de documents scientifiques de niveau recherche, publiés ou non, émanant des établissements d'enseignement et de recherche français ou étrangers, des laboratoires publics ou privés. 


\section{AIP | Physics of Fluids}

The bifurcation structure of viscous steady axisymmetric vortex breakdown with open lateral boundaries

Elena Vyazmina, Joseph W. Nichols, Jean-Marc Chomaz, and Peter J. Schmid

Citation: Physics of Fluids (1994-present) 21, 074107 (2009); doi: 10.1063/1.3176476

View online: http://dx.doi.org/10.1063/1.3176476

View Table of Contents: http://scitation.aip.org/content/aip/journal/pof2/21/7?ver=pdfcov

Published by the AIP Publishing

\section{Articles you may be interested in}

Open-loop control of noise amplification in a separated boundary layer flow

Phys. Fluids 25, 124106 (2013); 10.1063/1.4846916

Analysis of Reynolds number scaling for viscous vortex reconnection

Phys. Fluids 24, 105102 (2012); 10.1063/1.4757658

Control of axisymmetric vortex breakdown in a constricted pipe: Nonlinear steady states and weakly nonlinear asymptotic expansions

Phys. Fluids 23, 084102 (2011); 10.1063/1.3610380

Mechanics of viscous vortex reconnection

Phys. Fluids 23, 021701 (2011); 10.1063/1.3532039

Steady axisymmetric flow in an open cylindrical container with a partially rotating bottom wall

Phys. Fluids 17, 063603 (2005); 10.1063/1.1932664

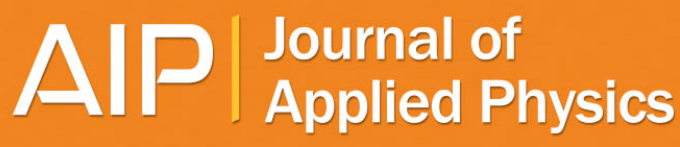

Journal of Applied Physics is pleased to announce André Anders as its new Editor-in-Chief 


\title{
The bifurcation structure of viscous steady axisymmetric vortex breakdown with open lateral boundaries
}

\author{
Elena Vyazmina, ${ }^{a}$ Joseph W. Nichols, Jean-Marc Chomaz, and Peter J. Schmid \\ Laboratoire d'Hydrodynamique (LadHyX), CNRS, École Polytechnique, 91128 Palaiseau Cedex, France
}

(Received 22 December 2008; accepted 28 May 2009; published online 31 July 2009)

\begin{abstract}
The effect of small viscosity on the behavior of the incompressible axisymmetric flow with open lateral and outlet boundaries near the critical swirling number has been studied by numerical simulations and asymptotic analysis. This work extends the theoretical studies of Wang and Rusak and numerical results of Beran and Culik to the case of flow with open lateral and outlet boundaries. In the inviscid limit the columnar flow state constitutes a solution that is known to become unstable at a particular swirl parameter. An asymptotic expansion shows that for small perturbations about this inviscid state an exchange of stability gives rise to a double saddle node bifurcation. The solution of the Euler equations breaks into two branches of the Navier-Stokes equations with a gap between the branches in which no near-columnar flow can exist. Around this region, two steady-state solutions exist for the same boundary conditions, one close to the columnar state and the other corresponding to either an accelerated or a decelerated state. This bifurcation structure is verified by numerical simulations, where the Navier-Stokes solutions are computed using branch continuation techniques based on the recursive projection method. For relatively small Reynolds numbers the numerically computed bifurcation curve does not exhibit any characteristic fold, and thus no hysteresis behavior. In this case, only a single equilibrium solution is found to exist, which changes monotonically from the quasicolumnar state to the breakdown solution. For large Reynolds numbers, however, the numerically determined bifurcation diagram confirms the fold structure characterized by the disappearance of the nearly columnar state via a saddle node bifurcation. Using the minimum axial velocity on the axis as a measure of the flow state we show that the agreement between theory and numerics is asymptotically good. (C) 2009 American Institute of Physics.
\end{abstract}

[DOI: 10.1063/1.3176476]

\section{INTRODUCTION}

Vortex breakdown is a feature of many flows that have both axial and azimuthal velocity components; these flow fields are known as swirling flows. It is characterized by an abrupt and dramatic change in the structure of the axisymmetric core, which leads to the appearance of stagnation points on or near the axis of symmetry followed by regions of reversed flow referred to as the vortex breakdown bubble.

The study of vortex breakdown is of great interest to, among other fields, aerodynamics and combustion physics. Breakdown also arises in a number of natural settings such as tornadoes, dust devils, and water spouts. ${ }^{1}$ Its occurrence in the flow over delta wings at high angles of attack can have a significant effect on lift, drag, and pitching moment as reported by Hummel and Srinivasan. ${ }^{2}$ For the design of combustion chambers, Beer and Chigier $^{3}$ and Faler and Leibovich ${ }^{4}$ emphasized the importance of understanding the flow structure of vortex breakdown. In this configuration, breakdown is intentionally triggered to improve air-fuel mixing, and thus produce a more stable and compact flame as well as a more complete combustion process.

Scientific interest in explaining this nonlinear phenomenon has produced a great body of experimental, numerical, and theoretical studies. In addition, several review articles on

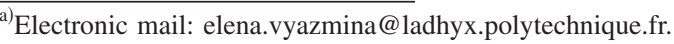

vortex breakdown have appeared: See, for example, Refs. 5-14. According to these reviews various stability criteria have been developed and proposed over the years. Despite a great deal of progress, many details of the vortex breakdown process are still poorly understood, and the continued study of this phenomenon is essential both for fundamental reasons and for the development of different technological devices such as hydrocyclone separators, ${ }^{15}$ combustion chambers, ${ }^{8}$ nozzles, and other applications where swirl plays an important role.

In an early study, Squire ${ }^{16}$ and Benjamin ${ }^{17}$ investigated inviscid, incompressible, axisymmetric, swirling flow in a pipe. They defined a critical level of swirl $S_{c B}$ when infinitely long small-amplitude axisymmetric standing waves appear in the flow. Supercritical vortex flow with a swirl of $S<S_{c B}$ does not support such waves, whereas subcritical flow with $S>S_{c B}$ does.

Leibovich $^{18}$ revealed that the critical state is a singular point for the inviscid steady flow. Using weakly nonlinear asymptotic analysis he observed a branch of the steady-state Euler equations that bifurcates at the critical swirl from the columnar state and continues into the region $S<S_{c B}$. This branch describes a standing solitary wave arising from the base columnar state in an infinitely long pipe.

Keller et al. ${ }^{19}$ considered inviscid axisymmetric vortex breakdown in an infinitely long pipe characterized by a semi- 


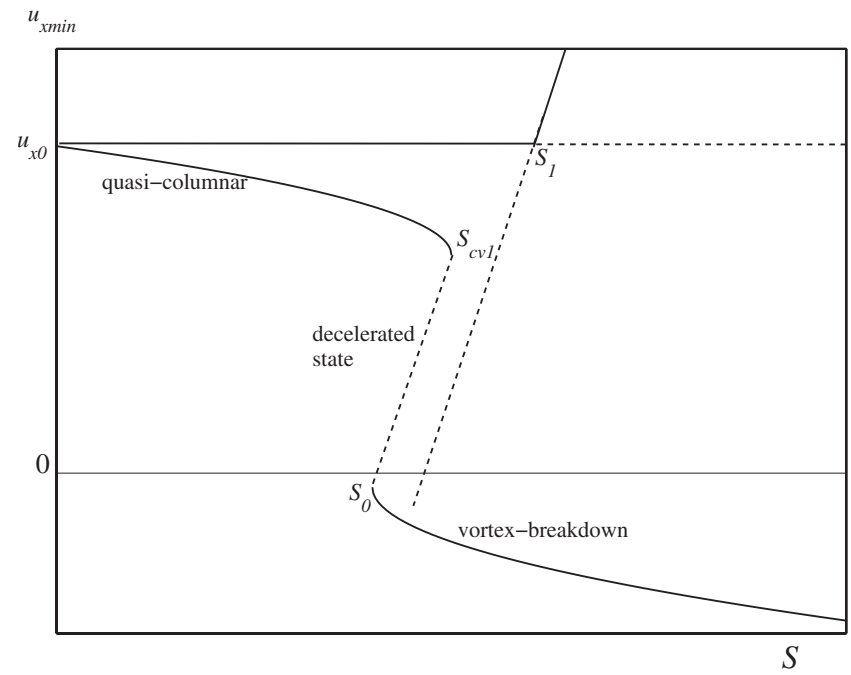

FIG. 1. Qualitative bifurcation diagram for axisymmetric vortex breakdown for both inviscid and viscous flows. Here, $u_{x \min }$ is the minimum axial velocity found at any point in the computational domain.

infinite stagnation region with free boundaries on the base columnar flow. This solution describes the transition from a base inlet columnar state to another columnar state further downstream that has the same dynamics due to the conservation of axial momentum along the pipe. For a fixed vortical core radius at the inlet, this solution only exists for a specific swirl $S_{0}$ with $S_{0}<S_{c B}$.

In a sequence of papers, Rusak and co-workers ${ }^{14,20-28}$ comprehensively investigated the dynamics and stability behavior of an incompressible axisymmetric vortex flow in a finite-length circular pipe. Fixed profiles for axial flow, circulation, and azimuthal vorticity have been imposed at the inlet of the pipe, together with zero-radial-velocity boundary conditions at the outlet. These conditions take into account the flow behavior observed in the experimental work of Bruecker and Althaus ${ }^{29}$ who reported that the inlet flow did not change appreciably as vortex breakdown occurred. The same behavior has also been demonstrated in the numerical investigations of Beran, ${ }^{30}$ Lopez, ${ }^{31}$ and Snyder and Spall. ${ }^{32}$ Based on these conditions the theoretical work of Rusak and co-workers provides an encompassing picture of the physical mechanisms underlying axisymmetric vortex breakdown (see Fig. 1). The existence of three steady branches connected by two critical levels of swirls $S_{0}$ and $S_{1}\left(S_{0}<S_{1}\right)$ has been shown, where the branch corresponding to the critical swirl $S_{1}$ represents an extension of Benjamin's ${ }^{17}$ theory of vortex breakdown in an infinite pipe to the case of a finite-length pipe, while the branch associated with $S_{0}$ is an extension of that of Keller et al. ${ }^{19}$ also to a finite-length pipe. As was reported by Wang and Rusak ${ }^{20}$ the columnar state is absolutely stable for $S<S_{0}$, linearly stable for $S_{0}<S<S_{1}$, and unstable for $S>S_{1}$. The solitary-wave branch connecting the states corresponding to the swirls $S_{0}$ and $S_{1}$ is unstable and describes axisymmetric traveling waves convecting downstream. The breakdown branch originating from the state with swirl $S_{0}$ is globally stable for any swirl $S>S_{0}$ (see Fig. 1).

The analysis of Wang and co-workers ${ }^{20-23}$ shows that the critical flow state at a swirl $S_{1}$ consists of a marginal equilibrium. Mathematically, it is well known that transcritical bifurcations, such as the one near swirl $S_{1}$, are structurally unstable, i.e., any perturbation to the solution near the critical point can lead to significant changes in the bifurcation behavior, and thus in the nature of the solution. ${ }^{21,22}$ When perturbed, the transcritical bifurcation at $S_{1}$ separates into two branches which no longer meet at $S_{1}$. For example, Lopez ${ }^{31}$ found a fold indicating the existence of multiple solutions as well as hysteresis and limit point behavior, characteristic of a perturbed transcritical bifurcation.

At present, most theoretical and numerical investigations have primarily focused on swirling flow in pipes with corresponding boundary conditions. In this paper we generalized the analysis of swirling flows to the case with open lateral and outflow boundaries. This work furthermore extends the asymptotic analysis of Wang and Rusak ${ }^{24}$ to this different set of lateral and outlet boundary conditions. While swirling flows in combustion chambers are confined, the geometry of these chambers is usually complex. Furthermore, vortex breakdown also occurs in applications where the flow is partially or fully unconfined such as the flow over delta wings or the geophysical flows mentioned above. Since both of these scenarios (complex confined geometry and unconfined geometry) have significantly different boundary conditions than those for flow in a pipe, we focus in the present paper on the influence of these boundary conditions on the vortex breakdown solutions obtained over the entire domain. This is accomplished by comparing results obtained with different boundary conditions using both an extended asymptotic analysis and direct numerical simulation (DNS).

The paper is organized as follows. Section II gives the equations governing viscous vortex breakdown and provides a detailed description of the chosen boundary conditions. In Sec. III the numerical simulations are presented. The critical swirl number is found in Sec. IV. The asymptotic analysis of near-critical swirling flow is provided in Sec. V. Then, in Sec. VI, the asymptotic and numerical solutions of the problem are compared and the relation between the present investigation and the breakdown of vortex flow in a pipe is discussed. Finally, our results are summarized in Sec. VII.

\section{MATHEMATICAL MODEL}

To model vortex breakdown, we consider an unsteady, axisymmetric, incompressible viscous flow of constant density $(\rho=1)$ in a cylindrical domain of outer radius $R$ and axial length $x_{0}$. We use cylindrical coordinates where $x, r$, and $\theta$ denote the axial, radial, and azimuthal directions, respectively. Likewise, the components of velocity in the axial, radial, and azimuthal directions are represented by $u_{x}, u_{r}$, and $u_{\theta}$, respectively, and $p$ denotes the pressure. We note that the ordering of the velocity components used in this paper differs from the convention used in Ref. 24.

The flow is governed by the axisymmetric NavierStokes and continuity equations which, in nondimensional form, read 


$$
\begin{aligned}
\frac{\partial u_{x}}{\partial t} & +u_{x} \frac{\partial u_{x}}{\partial x}+u_{r} \frac{\partial u_{x}}{\partial r}+\frac{\partial p}{\partial x} \\
& -\frac{1}{\operatorname{Re}}\left(\frac{\partial^{2} u_{x}}{\partial x^{2}}+\frac{\partial^{2} u_{x}}{\partial r^{2}}+\frac{1}{r} \frac{\partial u_{x}}{\partial r}\right)=0, \\
\frac{\partial u_{r}}{\partial t} & +u_{x} \frac{\partial u_{r}}{\partial x}+u_{r} \frac{\partial u_{r}}{\partial r}-\frac{u_{\theta}^{2}}{r}+\frac{\partial p}{\partial r} \\
& -\frac{1}{\operatorname{Re}}\left(\frac{\partial^{2} u_{r}}{\partial x^{2}}+\frac{\partial^{2} u_{r}}{\partial r^{2}}+\frac{1}{r} \frac{\partial u_{r}}{\partial r}-\frac{u_{r}}{r^{2}}\right)=0, \\
\frac{\partial u_{\theta}}{\partial t}+ & u_{x} \frac{\partial u_{\theta}}{\partial x}+u_{r} \frac{\partial u_{\theta}}{\partial r}+\frac{u_{r} u_{\theta}}{r} \\
& -\frac{1}{\operatorname{Re}}\left(\frac{\partial^{2} u_{\theta}}{\partial x^{2}}+\frac{\partial^{2} u_{\theta}}{\partial r^{2}}+\frac{1}{r} \frac{\partial u_{\theta}}{\partial r}-\frac{u_{\theta}}{r^{2}}\right)=0 \\
\frac{\partial u_{x}}{\partial x} & +\frac{1}{r} \frac{\partial}{\partial r}\left(r u_{r}\right)=0 .
\end{aligned}
$$

These equations have been nondimensionalized by a characteristic length equal to the radius of the vortex core, $r_{\text {core }}$, and a characteristic velocity taken as the inlet axial velocity, $u_{x 0}$. This results in a Reynolds number defined as

$$
\operatorname{Re}=\frac{u_{x 0} r_{\text {core }}}{\nu}
$$

where $\nu$ is the kinematic viscosity of the fluid. We have stated the time-dependent governing equations since our numerical simulations solve these equations by time stepping and recursive projection until a steady state is reached, as described in Sec. III. The steady vortex breakdown states of interest then satisfy the above system of governing equations with the time-dependent terms equal to zero.

At the inlet, the nondimensional axial, radial, and azimuthal velocity components are prescribed as

$$
\begin{aligned}
& u_{x}(0, r)=1, \\
& u_{r}(0, r)=0, \\
& u_{\theta}(0, r)=U_{\theta}\left(r^{\prime}\right) / u_{x 0}=S u_{\theta 0}(r),
\end{aligned}
$$

where $U_{\theta}$ is the dimensional azimuthal velocity profile in dimensional coordinates $r^{\prime}=r r_{\text {core }}$. The nondimensional swirl parameter $S=U_{\theta}\left(r_{\text {core }}\right) / u_{x 0}$ represents the ratio of the azimuthal velocity at the edge of the core to the axial freestream velocity. This definition of the swirl parameter enforces the normalization $u_{\theta 0}(1)=1$ of the nondimensional azimuthal velocity profile at the inlet. The total velocity profile given by Eq. (1) is axisymmetric and will be held constant at the inlet of our domain. Among the velocity profiles in the category described by Eq. (1) are the Burgers vortex that was used by, e.g., Beran and Culik ${ }^{33}$ and the Grabowski profile introduced by Grabowski and Berger ${ }^{34}$ and used recently by Ruith et al. ${ }^{35}$

At the outlet we apply Neumann boundary conditions for each velocity component as in Ref. 35,

$$
\frac{\partial u_{x}}{\partial x}\left(x_{0}, r\right)=0, \quad \frac{\partial u_{r}}{\partial x}\left(x_{0}, r\right)=0, \quad \frac{\partial u_{\theta}}{\partial x}\left(x_{0}, r\right)=0 .
$$

At the centerline we impose the conditions $u_{r}(x, 0)=0$ and $u_{\theta}(x, 0)=0$ due to the axisymmetry of the flow.

As emphasized by Ruith et $a l^{36}$ the use of free-slip boundary conditions in the radial direction requires excessively large computational domains to avoid backscatter from the radial boundaries. To truncate the domain at smaller radii, one must allow for mass and momentum to be exchanged across the radial boundary, and thus account for entrainment of exterior fluid into the jet. To this end, noviscous-traction boundary conditions in the radial direction, ${ }^{37}$

$$
\tau \cdot \boldsymbol{n}=0,
$$

are applied, where $\tau$ represents the viscous stress tensor and $\boldsymbol{n}$ stands for the unit normal vector in the lateral directions. In cylindrical coordinates this equation can be rewritten in component form as

$$
\begin{aligned}
& \frac{\partial u_{r}}{\partial r}(x, R)=0, \\
& \frac{\partial u_{r}}{\partial x}(x, R)+\frac{\partial u_{x}}{\partial r}(x, R)=0, \\
& \frac{\partial u_{\theta}}{\partial r}(x, R)-\frac{u_{\theta}}{r}(x, R)=0 .
\end{aligned}
$$

For our present investigation we neglect the fact that the inlet azimuthal velocity does not exactly satisfy the lateral boundary conditions (3). Following the argument given by Ruith et al., ${ }^{36}$ however, we note that for both the Grabowski and Burgers profiles the stress tensor component corresponding to the azimuthal velocity at the radial edge of the domain decays like $1 / R^{2}$, and therefore can be neglected for sufficiently large radial domains.

We remind the reader that the majority of past theoretical investigations used a no-flux radial boundary condition (reflecting the conservation of the total mass flux across the pipe) as well as a zero radial velocity at the outlet. In our study we analyze the vortex breakdown problem with open lateral boundary conditions and purely convective behavior at the outlet.

\section{NUMERICAL SIMULATIONS}

The numerical simulations are based on the incompressible time-dependent axisymmetric Navier-Stokes equations in cylindrical coordinates $(x, r, \theta)$. The computational domain has the dimensionless size $R=10$ and $x_{0}=20$; it is numerically resolved by $n_{r}=127$ and $n_{x}=257$ grid points in the radial and axial directions, respectively, with a uniform mesh in the axial direction and with an algebraic mapped mesh $^{38}$ in the radial direction which clusters grid points near the centerline and the lateral boundaries. To reach a steady state, simulations of the time-dependent Navier-Stokes equations 
were run until the $L_{2}$-norm of the difference of the velocity field from one time step to the next became smaller than $10^{-11}$.

The Grabowski profile ${ }^{34}$ is used for the radial velocity, and the axial and azimuthal velocity components are defined piecewise for the regions inside and outside a characteristic radius. The Grabowski profile represents a smooth change from solid body rotation inside the characteristic radius and potential flow farther away. The velocity profile at the inflow boundary is forced to be axisymmetric and constant over time,

$$
\begin{aligned}
& u_{x}(0, r)=1, \\
& u_{r}(0, r)=0, \\
& u_{\theta}(0,0 \leq r \leq 1)=S r\left(2-r^{2}\right), \\
& u_{\theta}(0,1 \leq r)=S / r .
\end{aligned}
$$

The outflow convective boundary conditions used in numerical computations,

$$
\frac{\partial u_{x}}{\partial t}+C \frac{\partial u_{x}}{\partial x}=0, \quad \frac{\partial u_{r}}{\partial t}+C \frac{\partial u_{r}}{\partial x}=0, \quad \frac{\partial u_{\theta}}{\partial t}+C \frac{\partial u_{\theta}}{\partial x}=0,
$$

which reduce, for the steady state, to Eq. (2) used in theory, were the same as in Ref. 36. The numerical simulations were carried out for zero normal viscous stress boundary conditions (3) on the lateral frontier of the domain.

The incompressible Navier-Stokes equations are solved by a pressure projection method. ${ }^{39}$ Spatial derivatives are approximated with sixth-order compact schemes, and a fourth-order Runge-Kutta scheme is used for integration in time. The code used in the present study was adapted from a code used to study nonswirling variable-density jets. For further details, please see Ref. 40.

As a representative reference case, a swirling jet is selected with the dimensionless governing parameters of Re $=200$ and $S=1.095$. This choice is identical to the reference cases obtained by Grabowski and Berger ${ }^{34}$ and by Ruith et al. ${ }^{35}$ This results in simulations that closely match the streamline patterns presented in Fig. 3, frames (a) and (b), of Ruith et al. ${ }^{35}$

The recursive projection method (RPM) of Shroff and Keller ${ }^{41}$ is used as a tool to stabilize the fixed-point iterative procedure and also as a convergence accelerator. RPM seeks to identify the space associated with the dominant eigenvalues and to eliminate its negative influence on the original fixed-point iteration by combining it with Newton iterations for the identified subspace. Once a steady state is found, the eigenvalues determined by the RPM procedure give directly its stability properties.

In order to demonstrate the bifurcation structure of the flow, a quantitative measure of the flow is needed to monitor the development of the steady-state solutions as the governing parameters are varied. An appropriate diagnostic quantity is $u_{x \min }$, the minimum of the axial velocity in the meridional half-plane, which is equivalent to the minimum of axial velocity in the entire domain. In the current investigation, two governing parameters are varied, the swirl parameter $S$ and the Reynolds number Re. For each choice of these parameters we compute a steady-state branch of the solution, where each new steady-state computation uses the previously calculated steady state as an initial condition.

In Fig. 2, the steady-state solution branch for $\mathrm{Re}=200$ represents the spatial evolution of streamlines dependent on the swirl parameter. In the figure, steady-state solutions were computed at 342 separate values of $S, u_{x \min }$ was extracted from each of these solutions, and the solid curve was plotted through these points using linear interpolation. Here, we observe the gradual change in the solution from the columnar state $(a)$ to vortex breakdown states $(d, e, f)$. From this bifurcation structure the development of recirculation bubbles can be studied. As the swirl increases, the appearance of a single recirculation bubble indicates the initial onset of vortex breakdown. In the figure, this occurs when the bifurcation curve passes through point $c$, where $u_{x \min }$ first becomes negative owing to the presence of recirculation. As the swirl parameter increases further yet, a second recirculation bubble forms just downstream of the first, as shown in state $f$.

In a similar fashion as in Fig. 2, Fig. 3 shows the steadystate solution branch for $\mathrm{Re}=1000$, together with the swirldependent spatial evolution of the streamlines. In this case, 373 separate steady-state solutions were computed, slightly more than in the previous case. More solutions were needed because of the small arc-length parameter necessary for continuation in the vicinity of the critical point labeled $b$ in Fig. 3 where the slope of the bifurcation curve becomes vertical. This critical point $(b)$ divides the stable columnar branch of the bifurcation curve from the unstable branch. The streamline pattern of solution state $a$ in Fig. 3 is representative of the branch corresponding to the columnar state, which is characterized by relatively large positive values of $u_{x \mathrm{~min}}$. Beyond the critical point $(b)$, perturbations of the flow propagate downstream and a subsequent generation of the recirculation bubble is observed close to the outlet, as shown by the streamline plots corresponding to solution states $d$ and $e$. This is in contrast to the calculations shown in Fig. 2 where the recirculation bubble forms closer to the inlet and means that the higher Reynolds number flow strongly interacts with outlet boundary conditions. Also in contrast to the previous calculations, where stable solutions are found along the entire bifurcation curve for $\operatorname{Re}=200$, solutions found beyond the critical point for $\mathrm{Re}=1000$ are linearly unstable. It is important to note, however, that the solutions become linearly unstable only beyond the critical point in the Re $=1000$ case, and so this branch is referred to as the unstable steady-state branch to distinguish it from the stable columnar branch. Initializing DNS with this unstable state, we observe an exponential departure away from the initial condition and eventual convergence to the stable columnar branch. In the case of pipe flow, the unstable steady-state branch connects two stable branches (columnar and vortex breakdown), which are part of one curve with a fold as described by Ref. 31 . For the case considered here we found the spatial struc- 

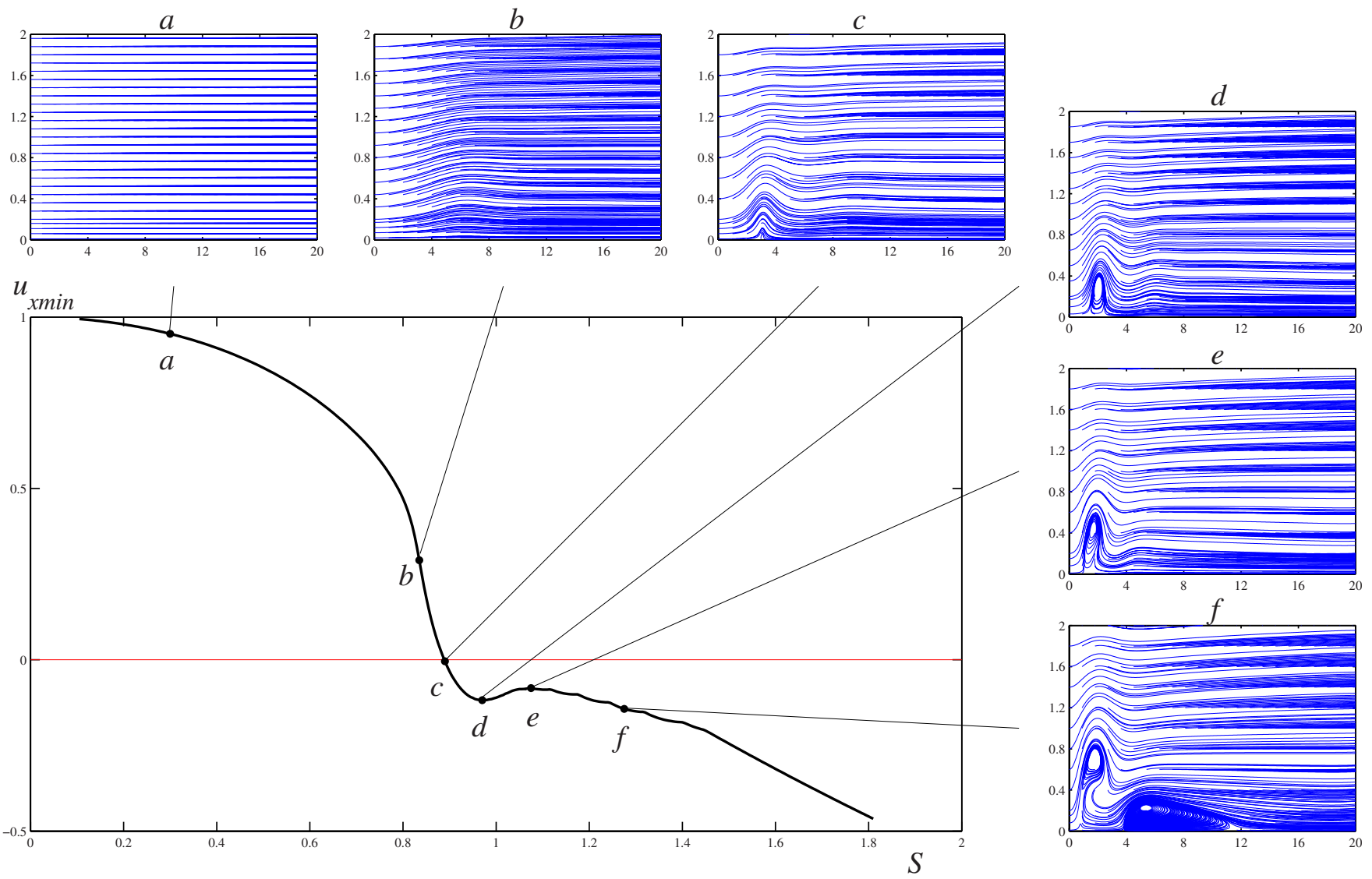

FIG. 2. (Color online) Bifurcation diagram describing the formation of vortex breakdown as the swirl is increased. The minimum axial velocity $u_{x \min }$ is plotted as a function of the swirl number $S$ for $\mathrm{Re}=200$. Each point along the bifurcation curve corresponds to a steady-state solution of the Navier-Stokes equations. The streamlines of some of the corresponding characteristic steady states are shown on the top and on the right. The wiggles on the bifurcation diagram visible after $S=1$ are converged (i.e., identical when the resolution is increased); they disappear when a less specific measure is taken as, for example, the overall mean value of $u_{x}$.

ture of the vortex breakdown branch to be highly sensitive to the swirl parameter when Reynolds numbers are high. This prevented us from following this entire branch and connecting it to the unstable one. In this region, a continuation increment of $10^{-5}$ or $10^{-6}$ on swirl parameter was needed to follow the bifurcation curve. In addition, each point along the curve required approximately $10^{5}$ iterations to converge to a steady solution; therefore, this would require a total of $10^{10}$ iterations to traverse the necessary range of swirl numbers. Small portions of the vortex breakdown branch have been computed exhibiting one (streamline pattern $f$, Fig. 3) or two bubbles depending on the swirl number value.

By examining the flow behavior (see the streamline patterns in Fig. 3) it is easy to conclude that the problem strongly depends on the outlet boundary conditions, i.e., the manner in which velocity perturbations leave the computational domain. The Neumann outlet boundary conditions allow an open recirculation region to exist. From a physical point of view this bubble comes from the outlet, and for a larger domain the vortex breakdown state will appear at smaller swirl parameters, and the recirculation bubbles will again form at the outlet in a similar way to Fig. 3.

\section{THE CRITICAL STATE OF INVISCID SWIRLING FLOW}

We consider a steady base flow given by an inviscid solution of the steady Euler equations which corresponds to transport downstream of the inlet boundary conditions (1). This base flow will be perturbed by infinitesimal disturbances as

$$
\begin{aligned}
& u_{x}(x, r)=u_{x 0}+\epsilon u_{x 1}(x, r)+\cdots, \\
& u_{r}(x, r)=\epsilon u_{r 1}(x, r)+\cdots, \\
& u_{\theta}(x, r)=S u_{\theta 0}(r)+\epsilon u_{\theta 1}(x, r)+\cdots, \\
& p(x, r)=p_{0}(r)+\epsilon p_{1}(x, r)+\cdots,
\end{aligned}
$$

where $\epsilon \ll 1$. Substitution into the Euler and continuity equations and considering only terms which are first order in $\epsilon$ results in the linearized equations 

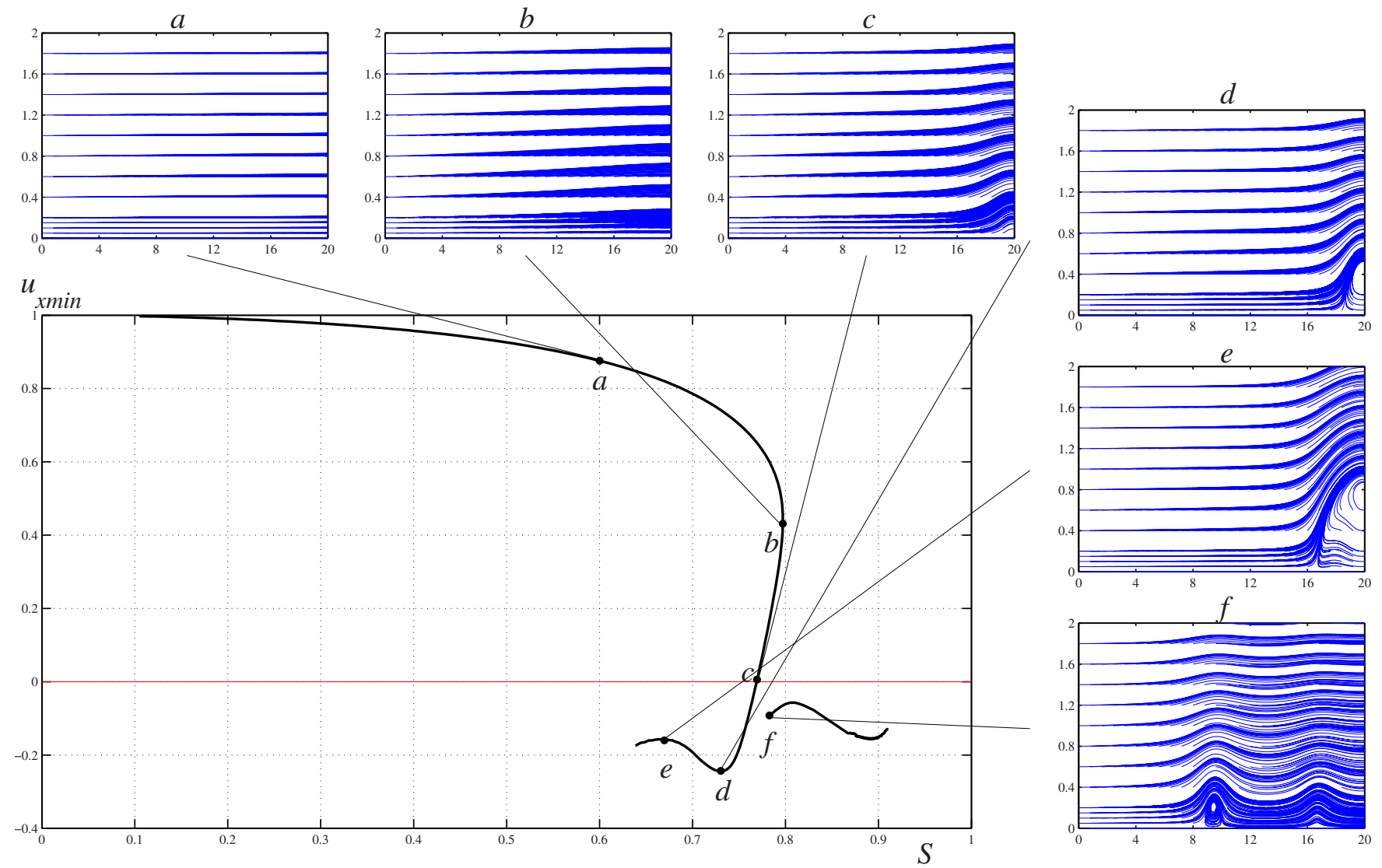

FIG. 3. (Color online) Bifurcation diagram describing the formation of vortex breakdown as the swirl is increased. The minimum axial velocity $u_{x \min }$ is plotted as a function of the swirl number $S$ for $\mathrm{Re}=1000$. Each point along the bifurcation curve corresponds to a steady-state solution of the Navier-Stokes equations. The streamlines of some of the corresponding characteristic steady states are shown on the top and on the right.

$$
\begin{aligned}
& u_{x 0} \frac{\partial u_{x 1}}{\partial x}+\frac{\partial p_{1}}{\partial x}=0 \\
& u_{x 0} \frac{\partial u_{r 1}}{\partial x}+\frac{\partial p_{1}}{\partial r}-2 \frac{u_{\theta 0} u_{\theta 1}}{r} S=0 \\
& u_{x 0} \frac{\partial u_{\theta 1}}{\partial x}+\frac{u_{r 1}}{r} \frac{\partial\left(r u_{\theta 0}\right)}{\partial r} S=0 \\
& \frac{\partial u_{x 1}}{\partial x}+\frac{1}{r} \frac{\partial\left(r u_{r 1}\right)}{\partial r}=0 .
\end{aligned}
$$

Eliminating pressure from the first two equations of (5) we arrive at

$$
\begin{aligned}
& u_{x 0} \frac{\partial^{2} u_{r 1}}{\partial x^{2}}-u_{x 0} \frac{\partial^{2} u_{x 1}}{\partial x r}-2 \frac{u_{\theta 0}}{r} S \frac{\partial u_{\theta 1}}{\partial x}=0, \\
& u_{x 0} \frac{\partial u_{\theta 1}}{\partial x}+\frac{u_{r 1}}{r} \frac{\partial\left(r u_{\theta 0}\right)}{\partial r} S=0, \\
& \frac{\partial u_{x 1}}{\partial x}+\frac{1}{r} \frac{\partial\left(r u_{r 1}\right)}{\partial r}=0 .
\end{aligned}
$$

Subsequent substitution of the continuity equation and the expression for $u_{\theta 1}$ into the first equation of Eq. (6) reduces system (5) to the linear partial differential equation for the radial velocity, $u_{r 1}$ :

$$
\begin{aligned}
& \frac{\partial}{\partial r}\left(\frac{1}{r} \frac{\partial r u_{r 1}}{\partial r}\right)+\frac{\partial^{2} u_{r 1}}{\partial x^{2}}+S^{2} \frac{2 u_{\theta 0} u_{r 1}}{r^{2} u_{x 0}^{2}} \frac{\partial\left(r u_{\theta 0}\right)}{\partial r}=0, \\
& u_{r 1}(x, 0)=0, \quad \frac{\partial u_{r 1}}{\partial r}(x, R)=0, \\
& u_{r 1}(0, r)=0, \quad \frac{\partial u_{r 1}}{\partial x}\left(x_{0}, r\right)=0 .
\end{aligned}
$$

Equation (7) can be rewritten in the form $L u_{r 1}=0$, where $L$ is a linear partial differential operator defined by the following expression:

$$
L=\frac{\partial}{\partial r}\left(\frac{1}{r} \frac{\partial}{\partial r} r\right)+\frac{\partial^{2}}{\partial x^{2}}+S^{2} \frac{2 u_{\theta 0}}{r^{2} u_{x 0}^{2}} \frac{\partial\left(r u_{\theta 0}\right)}{\partial r} .
$$

It is important to point out that this problem has nontrivial solutions only for specific values of $S$, corresponding to the bifurcation points. For other values of $S$, if a nonsteady linear solution was computed, it would have a growth rate different from zero.

The solution of Eq. (7) $u_{r 1}(x, r)=A \tilde{u}_{r 1}(x, r)$ is obtained by separation of variables according to 


$$
\tilde{u}_{r 1}(x, r)=\sin (\lambda x) \Phi(r),
$$

where $A$ denotes amplitude. The first eigenvalue $S_{1}$ of Eq. (7) defines the critical swirl of the flow. The critical swirl $S_{1}$ is a bifurcation point of solution branches of the steady Euler equation obtained by the above separation with $\lambda=\pi / 2 x_{0}$, where $\Omega_{1}=S_{1}^{2}$ and $\Phi(r)$ are determined by the eigenvalue problem

$$
\begin{aligned}
& \left.\frac{d}{d r}\left(\frac{1}{r} \frac{d(r \Phi(r))}{d r}\right)+\left(-\lambda^{2}+\Omega_{1} \frac{2 u_{\theta 0}}{r^{2} u_{x 0}^{2}} \frac{d\left(r u_{\theta 0}\right)}{d r}\right)\right] \Phi(r)=0, \\
& \Phi(0)=0, \quad \frac{d \Phi}{d r}(R)=0 .
\end{aligned}
$$

The associated perturbation velocities are

$$
\begin{aligned}
& \tilde{u}_{x 1}(x, r)=[\cos (\lambda x)-1] \frac{1}{\lambda} \frac{1}{r} \frac{d(r \Phi(r))}{d r}, \\
& \tilde{u}_{\theta 1}(x, r)=[\cos (\lambda x)-1] \frac{S_{1}}{\lambda u_{x 0}} \frac{\Phi(r)}{r} \frac{d\left(r u_{\theta 0}\right)}{d r} .
\end{aligned}
$$

Since we are solving the inviscid problem we do not enforce the lateral and outlet boundary conditions on the axial and azimuthal velocities deduced from $\widetilde{u}_{r 1}$.

\section{ASYMPTOTIC EXPANSION OF NEAR-CRITICAL SWIRLING FLOWS IN THE LARGE REYNOLDS NUMBER LIMIT}

For small viscosities and small departure from the inviscid critical swirl $S_{1}$ we consider the perturbation approach used by Wang and Rusak ${ }^{24}$ about the critical inviscid solution for $S_{1}$. They have shown that two small parameters have to be introduced measuring the viscosity and the closeness to the critical inviscid state $\Delta \Omega$ to maintain a uniformly valid solution in the neighborhood of the critical swirl. We let $\Omega=S^{2}$ and $\Omega_{1}=S_{1}^{2}$, and anticipating the dominant balance valid when perturbing a transcritical bifurcation, we set $\Omega=\Omega_{1}+\epsilon \Delta \Omega^{\prime}$ and $\nu=\epsilon^{2} \nu^{\prime}$ with $\Delta \Omega^{\prime}=O(1)$ and $\nu^{\prime}=O(1)$. We then assume a perturbed solution in the form

$$
\begin{aligned}
& u_{x}(x, r)=u_{x 0}+\epsilon u_{x 1}(x, r)+\epsilon^{2} u_{x 2}(x, r)+\cdots, \\
& u_{r}(x, r)=\epsilon u_{r 1}(x, r)+\epsilon^{2} u_{r 2}(x, r)+\cdots, \\
& u_{\theta}(x, r)=S u_{\theta 0}(r)+\epsilon u_{\theta 1}(x, r)+\epsilon^{2} u_{\theta 2}(x, r)+\cdots, \\
& p(x, r)=p_{0}(r)+\epsilon p_{1}(x, r)+\epsilon^{2} p_{2}(x, r)+\cdots,
\end{aligned}
$$

where $\epsilon \ll 1$. The perturbation variables $u_{x 1}, u_{r 1}$, and $u_{\theta 1}$ satisfy the following boundary conditions:

$$
\begin{aligned}
& u_{x 1}(0, r)=0, \quad u_{r 1}(0, r)=0, \quad u_{\theta 1}(0, r)=0, \\
& u_{r 1}(x, 0)=0, \quad u_{\theta 1}(x, 0)=0 . \\
& \frac{\partial u_{r 1}}{\partial r}(x, R)=0,
\end{aligned}
$$

$$
\frac{\partial u_{r 1}}{\partial x}\left(x_{0}, r\right)=0
$$

We enforce the same boundary conditions on the higherorder terms $u_{x 2}, u_{r 2}$, and $u_{\theta 2}$.

At leading-order $\epsilon$ we recover the linear equation (7) with $S^{2}=\Omega_{1}$, which can be formally written as $L u_{r 1}=0$, where $L$ is defined by Eq. (8). As described in Sec. IV the solution of this equation is

$$
u_{r 1}(x, r)=A \tilde{u}_{r 1}(x, r)=A \sin (\lambda x) \Phi(r),
$$

with $A$ as an arbitrary amplitude to be determined by compatibility equations at higher order, $\lambda=\pi / 2 x_{0}$, and $\Phi(r)$ as the solution of Eq. (9).

At second order the linearized operator $L$ applied to $u_{r 2}$ is forced by terms stemming from the lower-order solution

$$
\begin{aligned}
{\left[u_{x 0}\right.} & \left.\frac{\partial}{\partial r}\left(\frac{1}{r} \frac{\partial r u_{r 2}}{\partial r}\right)+u_{x 0} \frac{\partial^{2} u_{r 2}}{\partial x^{2}}+\Omega_{1} \frac{2 u_{\theta 0} u_{r 2}}{r^{2} u_{x 0}} \frac{\partial\left(r u_{\theta 0}\right)}{\partial r}\right] \\
= & -A^{2}\left[\left(-\frac{\tilde{u}_{r 1}}{r}+\widetilde{u}_{x 1} \frac{\partial}{\partial x}+\widetilde{u}_{r 1} \frac{\partial}{\partial r}\right)\left(\frac{\partial \widetilde{u}_{r 1}}{\partial x}-\frac{\partial \widetilde{u}_{x 1}}{\partial r}\right)\right. \\
& \left.-\frac{2 \widetilde{u}_{\theta 1}}{r} \frac{\partial \tilde{u}_{\theta 1}}{\partial x}+\frac{2 u_{\theta 0} \sqrt{\Omega_{1}}}{r u_{x 0}}\left(\widetilde{u}_{x 1} \frac{\partial \widetilde{u}_{\theta 1}}{\partial x}+\frac{\widetilde{u}_{r 1}}{r} \frac{\partial r \tilde{u}_{\theta 1}}{\partial r}\right)\right] \\
& -A \Delta \Omega^{\prime} \frac{2 u_{\theta 0} \tilde{u}_{r 1}}{r^{2} u_{x 0}} \frac{\partial\left(r u_{\theta 0}\right)}{\partial r}+\nu^{\prime} \Omega_{1} \frac{2 u_{\theta 0}}{r u_{x 0}} \frac{\partial}{\partial r}\left(\frac{1}{r} \frac{\partial\left(r u_{\theta 0}\right)}{\partial r}\right) .
\end{aligned}
$$

Here the first term in the right-hand side of the equation is not linear, being proportional to $A^{2}$, and corresponds to the transport of the perturbation by the perturbation. The second term is linear in $A$ and originates from the change in the linearized operator with swirl parameter. The last term is independent of $A$ and represents the effect of viscosity on the base flow.

This equation may be formally written as $L u_{r 2}=\sigma\left(\widetilde{u}_{x 1}, \widetilde{u}_{r 1}, \widetilde{u}_{\theta 1}, u_{\theta 0}\right)$. It is easy to show that Eq. (7) is self-adjoint with respect to the scalar product

$$
\left\langle u_{r}^{\prime} \mid u_{r}\right\rangle=\iint u_{r}^{\prime} u_{r} r d r d x .
$$

Using the compatibility condition (Fredholm alternative) to find $u_{r 2}$ we need the forcing $\sigma\left(\widetilde{u}_{x 1}, \widetilde{u}_{r 1}, \widetilde{u}_{\theta 1}, u_{\theta 0}\right)$ to be orthogonal to the kernel of the adjoint that reads here $\left\langle\widetilde{u}_{r 1} \mid \sigma\right\rangle$ $=0$, giving

$$
A^{2} M_{1}-A \Delta \Omega^{\prime} M_{2}+\nu^{\prime} M_{3}=0,
$$




$$
\begin{aligned}
M_{1}= & \int_{0}^{x_{0}} \int_{0}^{R}\left[\left(-\frac{\tilde{u}_{r 1}}{r}+\tilde{u}_{x 1} \frac{\partial}{\partial x}+\tilde{u}_{r 1} \frac{\partial}{\partial r}\right)\left(\frac{\partial \tilde{u}_{r 1}}{\partial x}-\frac{\partial \tilde{u}_{x 1}}{\partial r}\right)\right. \\
& \left.-\frac{2 \tilde{u}_{\theta 1}}{r} \frac{\partial \tilde{u}_{\theta 1}}{\partial x}\right] \tilde{u}_{r 1} r d r d x \\
& +\int_{0}^{x_{0}} \int_{0}^{R} \frac{2 u_{\theta 0} \sqrt{\Omega_{1}}}{r u_{x 0}}\left(\tilde{u}_{x 1} \frac{\partial \tilde{u}_{\theta 1}}{\partial x}+\frac{\tilde{u}_{r 1}}{r} \frac{\partial r \tilde{u}_{\theta 1}}{\partial r}\right) \tilde{u}_{r 1} r d r d x, \\
M_{2}= & -\int_{0}^{x_{0}} \int_{0}^{R} \frac{2 u_{\theta 0} \tilde{u}_{r 1}}{r^{2} u_{x 0}} \frac{\partial\left(r u_{\theta 0}\right)}{\partial r} \tilde{u}_{r 1} r d r d x,
\end{aligned}
$$$$
M_{3}=-\int_{0}^{x_{0}} \int_{0}^{R} \Omega_{1} \frac{2 u_{\theta 0}}{r u_{x 0}} \frac{\partial}{\partial r}\left(\frac{1}{r} \frac{\partial\left(r u_{\theta 0}\right)}{\partial r}\right) \tilde{u}_{r 1} r d r d x .
$$

Integration over $x$ leads to

$$
M_{1}=\frac{x_{0}^{2}}{\pi^{2} u_{x 0}^{2}} N_{1}, \quad M_{2}=\frac{x_{0}}{u_{x 0}} N_{2}, \quad M_{3}=\frac{4 \Omega_{1} x_{0}}{\pi u_{x 0}} N_{3},
$$

with

$$
\begin{aligned}
N_{1}= & -2\left(\pi-\frac{4}{3}\right) \Omega_{1} \int_{0}^{R} \frac{\partial}{\partial r}\left(u_{\theta 0} \frac{\partial\left(r u_{\theta 0}\right)}{\partial r}\right) \frac{\Phi^{3}}{r} d r+\frac{\pi^{2} u_{x 0}^{2}}{12 x_{0}^{2}} \int_{0}^{R}\left[(3 \pi-8) \Phi+3 \pi \Phi_{r} r\right] \Phi^{2} d r \\
& -u_{x 0}^{2}\left(\pi-\frac{4}{3}\right) \int_{0}^{R} \frac{\left(\Phi_{r} \Phi_{r r}-\Phi \Phi_{r r r}\right) r^{3}+\left(\Phi \Phi_{r r}+\Phi_{r}^{2}\right) r^{2}+3 \Phi \Phi_{r} r-4 \Phi^{2}}{r^{2}} \Phi d r, \\
N_{2}= & -\int_{0}^{R} \frac{u_{\theta 0}}{r} \frac{\partial\left(r u_{\theta 0}\right)}{\partial r} \Phi^{2} d r, \\
N_{3}= & -\int_{0}^{R} u_{\theta 0} \frac{\partial}{\partial r}\left(\frac{1}{r} \frac{\partial\left(r u_{\theta 0}\right)}{\partial r}\right) \Phi d r .
\end{aligned}
$$

For the Grabowski profile, where the axial flow is uniform and equal to $u_{x 0}=1$, it can be shown that $N_{1}$ and $N_{3}$ are positive.

Equation (11) has a real solution for $A$ if

$$
\left|\Delta \Omega^{\prime}\right| \geq 2 \frac{\sqrt{M_{1} M_{3}}}{\left|M_{2}\right|} \sqrt{\nu^{\prime}}=4 \frac{\sqrt{N_{1} N_{3}}}{\left|N_{2}\right|} \sqrt{\frac{\nu^{\prime} x_{0} \Omega_{1}}{\pi^{3} u_{x 0}}} .
$$

If $\left|\Delta \Omega^{\prime}\right|<2 \sqrt{M_{1} M_{3} \nu^{\prime}} /\left|M_{2}\right|$, Eq. (11) has no real solutions; as mentioned by Wang and Rusak, ${ }^{24}$ in this case no steady viscous solution exists near the critical point.

Close to the critical state, with condition (13) satisfied,

$$
\begin{aligned}
A & =\frac{\Delta \Omega^{\prime} M_{2} \pm \sqrt{\left(\Delta \Omega^{\prime}\right)^{2} M_{2}^{2}-4 \nu^{\prime} M_{1} M_{3}}}{2 M_{1}} \\
& =\frac{\Delta \Omega^{\prime} N_{2} \pm \sqrt{\left(\Delta \Omega^{\prime}\right)^{2} N_{2}^{2}-16 \nu^{\prime} x_{0} \Omega_{1} N_{1} N_{3} /\left(\pi^{3} u_{x 0}\right)}}{2 x_{0} N_{1} /\left(\pi^{2} u_{x 0}\right)} .
\end{aligned}
$$

Multiplying Eq. (13) by $\epsilon$ we get that $|\Delta \Omega|$ $=2 \sqrt{M_{1} M_{3} \nu} /\left|M_{2}\right|$, meaning that no solution exists between $S_{\mathrm{c} \nu 1}$ and $S_{\mathrm{c} \nu 2}$,

$$
\begin{aligned}
& S_{\mathrm{c} \nu 1}^{2}=\Omega_{1}-4 \frac{\sqrt{N_{1} N_{3}}}{\left|N_{2}\right|} \sqrt{\frac{\nu x_{0} \Omega_{1}}{\pi^{3} u_{x 0}}}, \\
& S_{\mathrm{c} \nu 2}^{2}=\Omega_{1}+4 \frac{\sqrt{N_{1} N_{3}}}{\left|N_{2}\right|} \sqrt{\frac{\nu x_{0} \Omega_{1}}{\pi^{3} u_{x 0}}},
\end{aligned}
$$

which defines a saddle fold bifurcation point of the steady axisymmetric Navier-Stokes solution. The value $S_{\mathrm{c} \nu 1}^{2}$ corresponds to the first viscous correction to the inviscid critical swirl. $^{24}$

Starting from Eqs. (10), (9), and (14), multiplying by $\epsilon$, and neglecting all terms of higher order, the asymptotic expansion near the critical swirl $\Omega_{1}$ reads

$$
\begin{aligned}
& u_{x}(x, r)=u_{x 0}+\frac{\Delta \Omega N_{2} \pm \sqrt{(\Delta \Omega)^{2} N_{2}^{2}-16 \nu x_{0} \Omega_{1} N_{1} N_{3} /\left(\pi^{3} u_{x 0}\right)}}{2 x_{0} N_{1} /\left(\pi^{2} u_{x 0}\right)}[\cos (\lambda x)-1] \frac{1}{\lambda} \frac{1}{r} \frac{\partial[r \Phi(r)]}{\partial r}, \\
& u_{r}(x, r)=\frac{\Delta \Omega N_{2} \pm \sqrt{(\Delta \Omega)^{2} N_{2}^{2}-16 \nu x_{0} \Omega_{1} N_{1} N_{3} /\left(\pi^{3} u_{x 0}\right)}}{2 x_{0} N_{1} /\left(\pi^{2} u_{x 0}\right)} \sin (\lambda x) \Phi(r),
\end{aligned}
$$




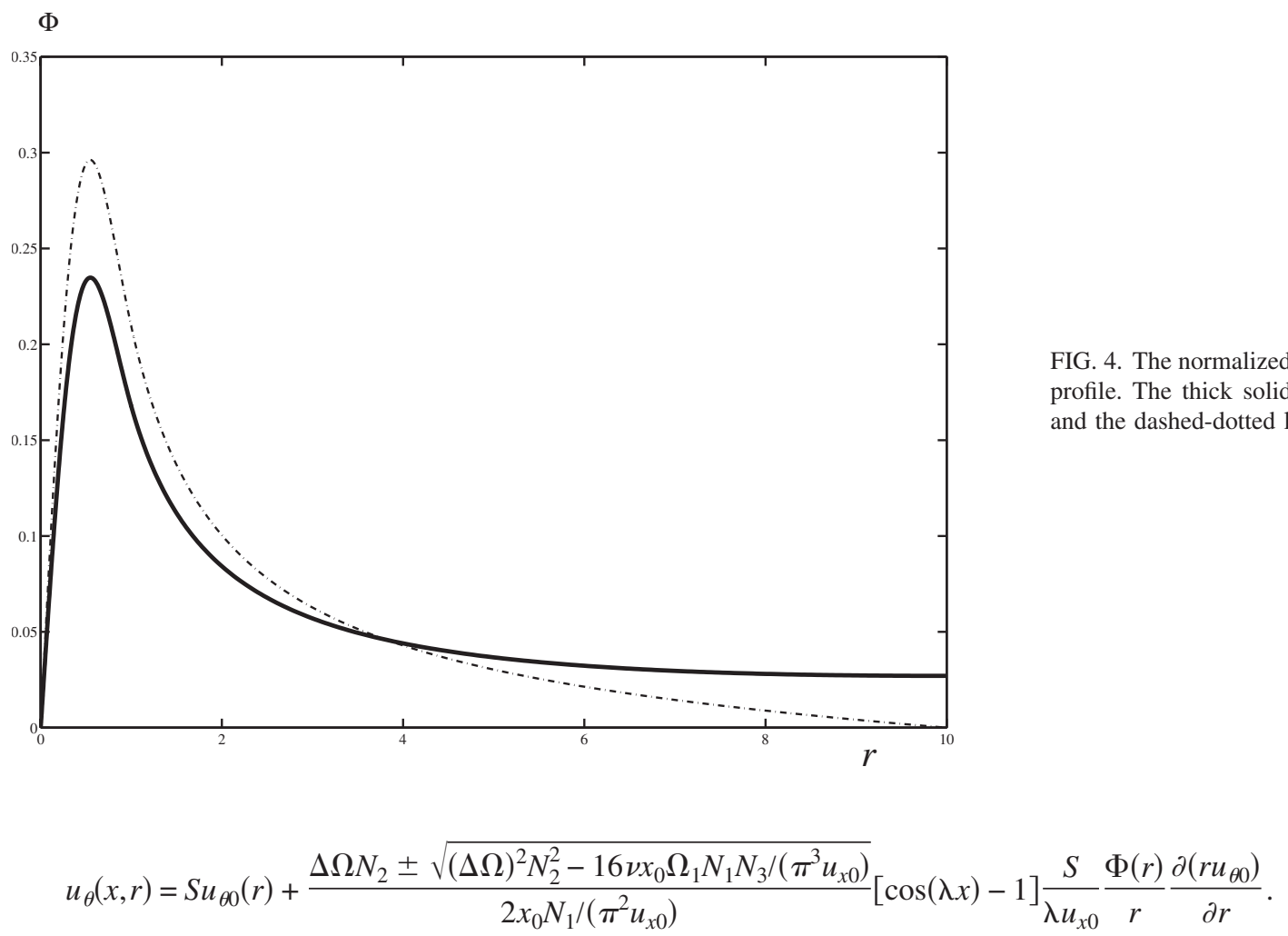

Careful analytical investigation of the expression for the axial velocity [Eq. (15)] shows that the minimum axial velocity always occurs at the centerline of the domain at the outlet for the decelerated state. Thus, the minimum axial velocity in the whole domain $u_{x \min }$ is equivalent to $u_{x}\left(x_{0}, 0\right)$.

We conclude, as in the pipe flow considered by Wang and Rusak, ${ }^{24}$ that, for a small but finite viscosity, the modified transcritical bifurcation of the Euler solution consists of two Navier-Stokes branches about $\Omega_{1}$ with a finite gap between these two branches equal to $8\left(\sqrt{N_{1} N_{3}} /\left|N_{2}\right|\right) \sqrt{\nu x_{0} \Omega_{1} / \pi^{3} u_{x 0}}$.

The bifurcation diagram in terms of the minimum axial velocity $u_{x \min }$ along the centerline, evaluated by using Eq. (15), is a nonlinear function in $\Delta \Omega$. Steady columnar flow at leading order exists for $S^{2}<S_{\mathrm{c} \nu 1}^{2}$ and $S^{2}>S_{\mathrm{c} \nu 2}^{2}$. The branches are not connected and the resulting gap near the critical swirl demonstrates that no near-columnar axisymmetric state exists for the corresponding range of the swirl parameter. Outside this region two near-columnar equilibrium states can exist for the same boundary conditions. For $S^{2}<S_{\mathrm{c} \nu 1}^{2}$ one branch describes a nearly columnar state and the other a decelerated axial flow which corresponds to the unstable steady-state branch described in Sec. III. The deceleration is evident in the streamline plots of Fig. 3, since the streamlines diverge as the flow develops downstream in this case. For $S^{2}>S_{\mathrm{c} \nu 2}^{2}$ one branch consists of the accelerated state and the second relaxes toward the columnar state.

\section{GRABOWSKI PROFILE}

For the Grabowski profile [Eq. (4)] we compute the eigenfunction $\Phi$ to determine the bifurcation behavior based on our asymptotic results. A spectral method based on Chebyshev polynomials (see, e.g., Ref. 42) was used to solve Eq. (9) resulting in the eigenfunction $\Phi(r)$ displayed in Fig. 4 by the solid thick line. Equation (9) outside the characteristic radius $r=1$ can be solved analytically, which gives the exact value $\Phi(R)=0.02704144306$ once the equation has been integrated in the core. The numerical integration in this outer region produces $\Phi(R)=0.027041443046$, which allows us to estimate the numerical error to be on the order of $10^{-11}$. The constants $N_{1}=0.059819851197575, \quad N 2$ $=-0.070295010313843$, and $N_{3}=0.763981542125681$ were computed using the Clenshaw-Curtis quadrature to approximate the integral equations (12) (see, e.g., Ref. 43).

To test the validity of these asymptotic results, we compare them to the results obtained from numerical simulations (Sec. III). Figure 5 compares the same solution branch for Reynolds numbers $\mathrm{Re}=2000$ and $\mathrm{Re}=1500$ obtained from numerical simulations (dashed thick and thin lines, respectively) and the asymptotic solution (solid lines of corresponding thickness). The straight black lines represent bifurcation curves for the inviscid case, and the intersection of the two straight lines defines the inviscid critical swirl number $S_{1}$. Perturbations increase as the Reynolds number decreases, which qualitatively agrees with the numerical results. ${ }^{44} \mathrm{~A}$ 
$u_{x \min }$

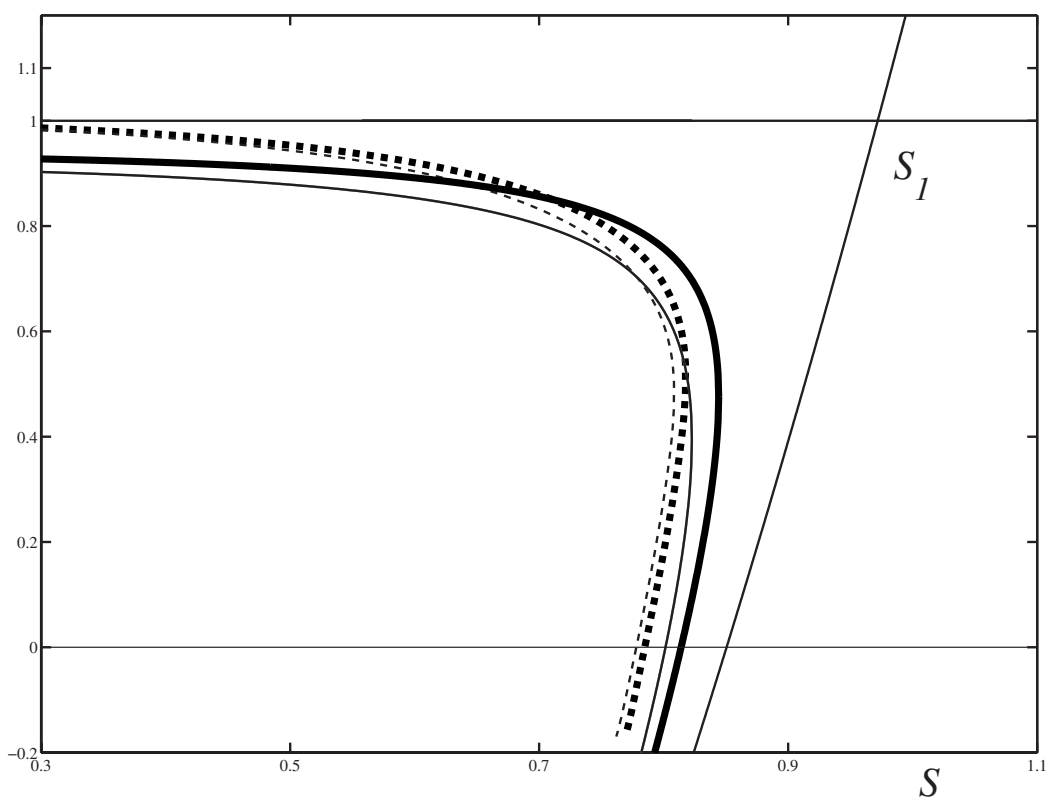

FIG. 5. Bifurcation curves obtained from asymptotic analysis for $\mathrm{Re}=1500$ (thin solid line) and $\mathrm{Re}=2000$ (thick solid line) and from numerical simulations for $\mathrm{Re}=1500$ (thin dashed line) and $\mathrm{Re}=2000$ (thick dashed line). similar dependence of the solution on the Reynolds number has already been discussed for the pipe case. ${ }^{24}$

Despite a Reynolds number of only $\mathrm{Re}=2000$, we obtain a good agreement between numerical calculations and asymptotic analysis from Sec. V. We expect an even closer match between the analytic and numerical bifurcation curves for higher Reynolds numbers; this attempt, however, would require a significantly larger resolution (i.e., denser computational grid, which in turn necessitates a smaller time step and an increased number of iterations to obtain the steadystate solution); the central processing unit time to calculate stable and unstable branches would be computationally too expensive.

Since we have verified that the extended analysis agrees well with the numerical simulations for $\mathrm{Re}=2000$, we may now use this tool to explore the effects of differing conditions at lateral and outlet boundaries. First, we compare our problem with open lateral boundaries to the flow in a pipe in which the lateral boundary condition is changed from an open, traction-free condition to a closed, free slip condition:

$$
u_{r 1}(x, R)=0,
$$

while keeping the same outlet (Neumann) boundary conditions.

The eigenfunction $\Phi(r)$ corresponding to this case is shown in Fig. 4 by the dashed-dotted line. One can observe a moderate difference between the two curves, but they have roughly the same shape. Even though the behavior of the curves near the lateral boundary at $r=10$ is different (the solid curve remains nonzero allowing entrainment whereas

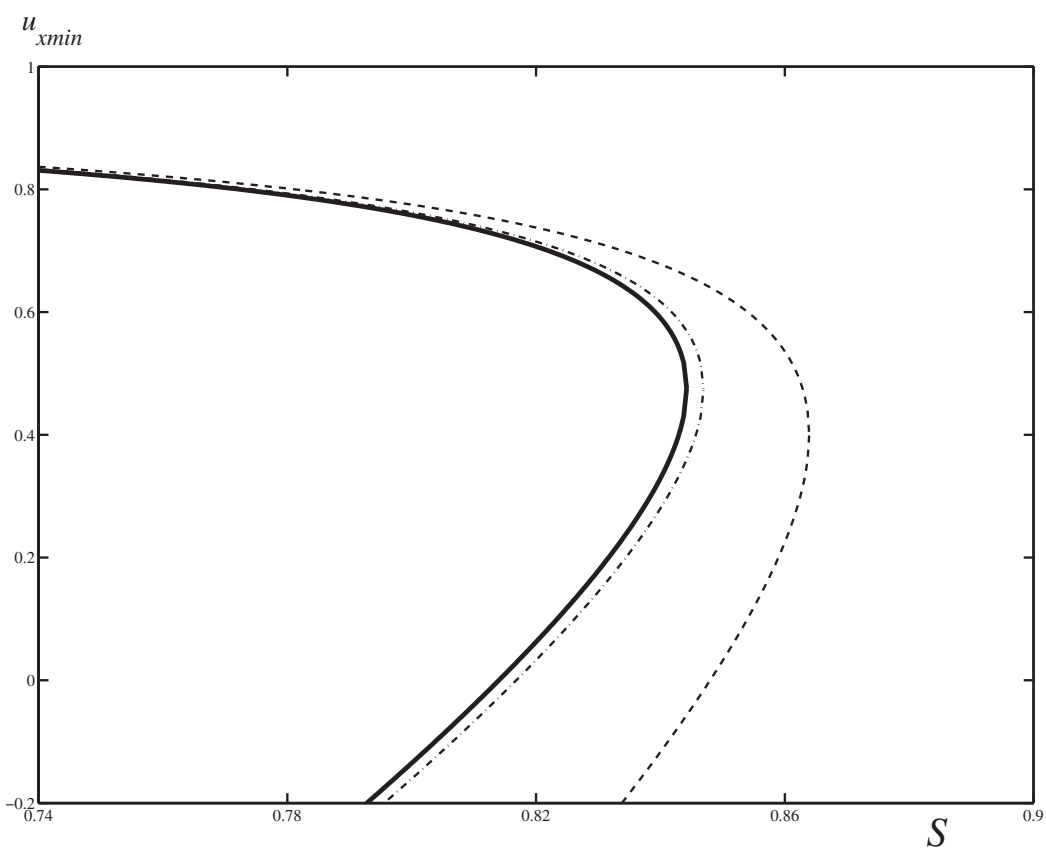

FIG. 6. Bifurcation curves obtained from asymptotic analysis for $\operatorname{Re}=2000$. The solid black line corresponds to the Neumann outlet problem with open lateral boundaries, the dashed-dotted line to flow in a pipe opened at the outlet, and the dashed curve to a flow in a pipe with zero radial velocity at the outlet (Ref. 24). 
the dashed-dotted curve does not), the peaks of the curves, near the center of the domain, are of about the same height and width. This indicates that the problem is only weakly sensitive to the type of imposed lateral boundary conditions.

The bifurcation curves corresponding to open and closed lateral boundaries (Fig. 6) also demonstrate that the solution depends very weakly on the lateral boundary conditions. Figure 6 also reports results obtained by Wang and Rusak ${ }^{24}$ on a pipe flow with a different outlet condition (Dirichlet). The difference is larger than when the lateral boundary condition alone is modified. This demonstrates a higher sensitivity to the outlet boundary condition. We found that the critical inviscid swirl number in the present case is $\Omega_{1}=0.94463073$, which is smaller than for a pipe with a Dirichlet boundary condition at the outlet, where $\Omega_{1}=0.95064678$, as reported by Wang and Rusak. ${ }^{24}$

\section{DISCUSSION AND CONCLUSIONS}

In this paper we investigated the influence of a small but finite viscosity on the bifurcation diagram of axisymmetric swirling Euler flow with traction-free lateral and convective outlet boundary conditions. We study the flow stability by investigating the bifurcation structure of steady-state solutions to the above problem. This has been accomplished both by means of numerical simulations and by theoretical analysis.

To validate our numerical simulations, we first considered the bifurcation structure of a low Reynolds number case $(\operatorname{Re}=200)$ as shown in Fig. 2. For this case, our computed steady-state solutions agreed very well with those found in the literature. ${ }^{35}$ Figure 2 shows that for small Reynolds numbers only one equilibrium solution exists, which represents the smooth monotonic change from the near-columnar state to vortex breakdown.

Numerical simulations and an asymptotic expansion about the critical swirl parameter for higher Reynolds numbers were also developed. The asymptotic analysis was carried out in a similar manner to Wang and $\mathrm{Rusak}^{24}$ but for different boundary conditions, namely, open lateral and outlet boundaries. As discussed in Sec. I, such a set of boundary conditions allows us to expand the theory of the swirling flows involving vortex breakdown to flow configurations such as combustion chambers, delta wings, and many others. It was shown that in a neighborhood of $S_{1}$ small but finite viscosity causes the steady Euler solution to give rise to two steady Navier-Stokes solutions whose branches show a gap. A small-disturbance analysis revealed a dependence on both viscosity as well as on a measure of the closeness to the critical swirl. It showed the existence of two critical viscous thresholds in parameter space, such that $S_{\mathrm{c} \nu 1}^{2}<S_{1}^{2}<S_{\mathrm{c} \nu 2}^{2}$, with the size of the gap $\left(S_{\mathrm{c} \nu 1}^{2}-S_{\mathrm{c} \nu 2}^{2}\right)$ proportional to $\sqrt{\nu x_{0} S_{1}^{2}}$. This means that no quasicolumnar states exist for $S_{\mathrm{c} \nu 1}^{2}<S_{1}^{2}$ $<S_{\mathrm{c} \nu 2}^{2}$. Experimental and numerical investigations conducted in this parameter range should obtain only one equilibrium solution: the vortex breakdown state. Outside this parameter range, however, up to three equilibrium states (quasicolumnar, decelerated or accelerated, and vortex breakdown) exist for identical boundary conditions and sufficiently large Rey- nolds numbers, as shown in Fig. 1. In this case, the decelerated state represents an unstable steady state and lies between the two other (columnar and vortex breakdown) states which are stable.

In spite of the fact that the inlet azimuthal velocity does not exactly satisfy the lateral boundary conditions, the present asymptotic analysis displayed good agreement with numerics, as shown in Fig. 5. Also, both the numerical and theoretical investigations found that the flow near the critical swirl is more sensitive to the outlet boundary conditions than to the lateral ones. Figure 6 shows that the asymptotic results changed more appreciably when the outlet boundary condition changed from Neumann to Dirichlet, in contrast to a change in the lateral boundaries from open to closed. From Fig. 3, we interpret the sensitivity of the solution to the outlet boundary condition to be caused by the nucleation of a recirculation bubble at the outlet boundary.

Asymptotic analysis also predicts the existence of an upper breakdown-free state for $S>S_{\mathrm{c} \nu 2}$, corresponding to the accelerated state. ${ }^{24}$ We wish to point out, however, that the viscous corrections in expansion (10) are valid for $\epsilon \ll \mid S_{2}$ $-S_{1} \mid$, where $S_{2}$ is the second eigenvalue of problem (9). In our case $(\operatorname{Re}=2000)$ these values are of the same order, i.e., $\epsilon \sim\left|S_{2}-S_{1}\right| \sim 0.02$, indicating that $\operatorname{Re}=2000$ is a too small Reynolds number to apply the theory for $S>S_{\mathrm{cv} 2}$, meaning that the upper fold might not exist for such a moderate Reynolds number. As mentioned above, we expect a better match between the analytic and numerical solutions as the Reynolds number increases.

\section{ACKNOWLEDGMENTS}

The authors would like to thank François Gallaire for helpful discussions throughout the course of this work. Furthermore, the authors thank the anonymous referees for their valuable comments. Financial support from DGA is gratefully acknowledged.

${ }^{1}$ L. N. Gutman, "Theoretical models of waterspout," Bull. Acad. Sci. USSR, Geophys. Ser. 1, 87 (1957).

${ }^{2}$ D. Hummel and P. S. Srinivasan, "Vortex breakdown effects on the lowspeed aerodynamic characteristics of slender delta wings in symmetrical flow," J. R. Aeronaut. Soc. 71, 319 (1967).

${ }^{3}$ J. Beer and N. Chigier, Combustion Aerodynamics (Applied Science, London, 1972).

${ }^{4}$ J. H. Faler and S. Leibovich, "An experimental map of the internal structure of vortex breakdown," J. Fluid Mech. 86, 313 (1978).

${ }^{5}$ M. G. Hall, "Vortex breakdown," Annu. Rev. Fluid Mech. 4, 195 (1972).

${ }^{6}$ S. Leibovich, "The structure of vortex breakdown," Annu. Rev. Fluid Mech. 10, 221 (1978).

${ }^{7}$ S. Leibovich, "Vortex stability and breakdown: Survey and extension," AIAA J. 22, 1192 (1984).

${ }^{8}$ M. Escudier, "Vortex breakdown: Observations and explanations," Prog. Aerosp. Sci. 25, 189 (1988).

${ }^{9}$ J. M. Delery, "Aspects of vortex breakdown," Prog. Aerosp. Sci. 30, 1 (1994).

${ }^{10}$ T. Sarpkaya, "Vortex breakdown and turbulence," AIAA Paper No. 950433, 1995.

${ }^{11}$ R. L. Ash and M. R. Khorrami, in Fluid Vortices, edited by S. I. Green (Kluwer, Boston, 1995), p. 317.

${ }^{12}$ W. Althaus, Ch. Bruecker, and M. Weimer, "Breakdown of slender vortices," in Fluid Vortices, edited by S. I. Green (Kluwer, Boston, 1995), p. 373.

${ }^{13}$ Z. Rusak and S. Wang, "Review of theoretical approaches to the vortex breakdown phenomenon," AIAA Paper No. 96-2126, 1996. 
${ }^{14} \mathrm{Z}$. Rusak, "Review of recent studies on the axisymmetric vortex breakdown phenomenon," AIAA Paper No. 2000-2529, 2000.

${ }^{15}$ T. Husveg, O. Rambeau, T. Drengstig, and T. Bilstad, "Performance of a decoiling hydrocyclone during variable flow rates," Minerals Eng. 20, 368 (2007).

${ }^{16}$ H. B. Squire, "Analysis of the vortex breakdown phenomenon," in Miszallaneen der angewandten Mechanik (Akademie, Berlin, 1960), pp. 306-312.

${ }^{17}$ T. B. Benjamin, "Theory of the vortex breakdown phenomenon," J. Fluid Mech. 14, 593 (1962)

${ }^{18}$ S. Leibovich, "Weakly nonlinear waves in rotating fluids," J. Fluid Mech. 42, 803 (1970).

${ }^{19}$ J. J. Keller, W. Egli, and W. Exley, "Force- and loss-free transitions between flow states," ZAMP 36, 854 (1985).

${ }^{20} \mathrm{~S}$. Wang and Z. Rusak, "The dynamics of swirling flow in a pipe and transition to axisymmetric vortex breakdown," J. Fluid Mech. 340, 177 (1997).

${ }^{21} \mathrm{~S}$. Wang and Z. Rusak, "On the stability of an axisymmetric rotating flow," Phys. Fluids 8, 1007 (1996).

${ }^{22}$ S. Wang and Z. Rusak, "On the stability of noncolumnar swirling flow," Phys. Fluids 8, 1017 (1996).

${ }^{23}$ Z. Rusak, S. Wang, and C. H. Whiting, "The evolution of a perturbed vortex in a pipe to axisymmetric vortex breakdown," J. Fluid Mech. 366, 211 (1998)

${ }^{24}$ S. Wang and Z. Rusak, "The effect of slight viscosity on near critical swirling flow," Phys. Fluids 9, 1914 (1997).

${ }^{25}$ Z. Rusak, K. P. Judd, and S. Wang, "The effect of small pipe divergence on near critical swirling flow," Phys. Fluids 9, 2273 (1997).

${ }^{26}$ Z. Rusak, C. H. Whiting, and S. Wang, "Axisymmetric breakdown of a Q-vortex in a pipe," AIAA J. 36, 1848 (1998).

${ }^{27}$ Z. Rusak, "The interaction of near critical swirling flows in a pipe with inlet vorticity perturbations," Phys. Fluids 10, 1672 (1998).

${ }^{28} \mathrm{Z}$. Rusak and K. P. Judd, "The stability of noncolumnar swirling flow in diverging streamtubes," Phys. Fluids 13, 2835 (2001).

${ }^{29} \mathrm{Ch}$. Bruecker and W. Althaus, "Study of vortex breakdown by particle tracking velocimetry (PTV), Part 3: Time-dependent structure and development of breakdown modes," Exp. Fluids 18, 174 (1995).
${ }^{30} \mathrm{P}$. S. Beran, "The time-asymptotic behavior of vortex breakdown in tubes," Comput. Fluids 23, 913 (1994).

${ }^{31}$ J. M. Lopez, "On the bifurcation structure of axisymmetric vortex breakdown in a constricted pipe," Phys. Fluids 6, 3683 (1994).

${ }^{32}$ D. O. Snyder and R. E. Spall, "Numerical simulation of bubble-type vortex breakdown within a tube-and-vane apparatus," Phys. Fluids 12, 603 (2000).

${ }^{33}$ P. S. Beran and F. E. S. Culik, "The role of non-uniqueness in the development of vortex breakdown in tubes," J. Fluid Mech. 242, 491 (1992).

${ }^{34}$ W. Grabowski and S. Berger, "Solutions for Navier-Stokes equations for vortex breakdown," J. Fluid Mech. 75, 525 (1976).

${ }^{35}$ M. R. Ruith, P. Chen, E. Meiburg, and T. Maxworthy, "Three-dimensional vortex breakdown in swirling jets and wakes: Direct numerical simulation," J. Fluid Mech. 486, 331 (2003).

${ }^{36}$ M. R. Ruith, P. Chen, and E. Meiburg, "Development of boundary conditions for direct numerical simulations of three-dimensional vortex breakdown phenomena in semi-infinite domains," Comput. Fluids 33, 1225 (2004).

${ }^{37}$ B. Boersma, G. Brethouwer, and F. Nieuwstadt, "A numerical investigation on the effect of the inflow conditions on the self-similar region of a round jet," Phys. Fluids 10, 899 (1998).

${ }^{38}$ A. Hanifi, P. J. Schmid, and D. S. Henningson, "Transient growth in compressible boundary layer flow," Phys. Fluids 8, 826 (1996).

${ }^{39}$ A. J. Chorin, "A numerical method for solving incompressible viscous flow problems," J. Comput. Phys. 2, 12 (1967).

${ }^{40}$ J. W. Nichols, P. J. Schmid, and J. J. Riley, "Self-sustained oscillations in variable-density round jets," J. Fluid Mech. 582, 341 (2007).

${ }^{41}$ G. M. Shroff and H. B. Keller, "Stabilisation of unstable procedures: The recursive projection method," SIAM (Soc. Ind. Appl. Math.) J. Numer. Anal. 30, 1099 (1993).

${ }^{42}$ P. J. Schmid and D. S. Henningson, Stability and Transition in Shear Flows (Springer-Verlag, New York, NY, 2001).

${ }^{43}$ L. N. Trefethen, Spectral Methods in Matlab (SIAM, Philadelphia, PA, 2000).

${ }^{44}$ E. Vyazmina, J. Nichols, J.-M. Chomaz, and P. Schmid, "Bifurcation structure of viscous vortex breakdown," AIAA Paper No. 2008-3797, 2008 